\title{
Procedimento Recursivo do Método dos Elementos de Contorno Aplicado em Problemas de Poisson
}

\author{
Vinicius Erler de Sousa Ramos ${ }^{1}$ \\ Instituto Federal de Educação, Ciência e Tecnologia do Espírito Santo, São Mateus, ES \\ Carlos Friedrich Loeffler ${ }^{2}$ \\ Programa de Pós Graduação em Engenharia Mecânica, PPGEM/UFES, Vitória, ES
}

\begin{abstract}
Resumo. Este trabalho apresenta um procedimento simples para melhorar a precisão dos valores numéricos obtidos com o Método dos Elementos de Contorno na solução de problemas de Poisson usando malhas com reduzido grau de refinamento. Usualmente, os valores em pontos internos são determinados através da reaplicação de uma equação integral, após serem calculados todos os valores de contorno. Neste trabalho, mostra-se que a mesma ideia pode ser utilizada para melhorar a precisão do método, escolhendo-se novos pontos fonte no contorno e reutilizando a equação integral de contorno.

Palavras-chave. Método dos Elementos de Contorno, Procedimento Recursivo, Método dos Resíduos Ponderados, Equação de Poisson
\end{abstract}

\section{Introdução}

A partir de observações obtidas pela precisão superior dos valores numéricos calculados com o Método dos Elementos de Contorno para pontos internos, em que a equação integral é novamente utilizada, propôs-se o estabelecimento do denominado procedimento recursivo, em que a equação integral é usada uma vez mais, com o propósito de melhorar a precisão dos resultados, recalculando-os em novos pontos fonte situados no contorno. A base matemática provém da ideia do Método dos Resíduos Ponderados (MRP), que é uma importante ferramenta usada para resolver numericamente equações diferenciais [1], baseado na minimização de resíduos. Em sua forma mais clássica, MRP usa funções de teste, para realizar aproximação de domínio global, em oposição com as estratégicas do Método dos Elementos de Contorno (MEC) ou Método dos Elementos Finitos (MEF), que usam aproximações setoriais, ou seja, funções de aproximação são usadas em partes limitadas. No entanto, a base matemática de MRP é mais geral, o que permite que sejam deduzidos os citados métodos e ainda

\footnotetext{
${ }^{1}$ vinicius.ramos@ifes.edu.br

2 carlosloeffler@bol.com.br
} 
outros métodos numéricos importantes, a partir da sentença fundamental do MRP [5], desde que as particularidades de cada técnica sejam adequadamente impostas.

O procedimento recursivo já foi aplicado com êxito em problemas de Laplace [4]. Neste trabalho, a técnica é aplicada aos problemas de Poisson. Os resultados numéricos são comparados com os analíticos para mostrar as melhorias obtidas com a técnica proposta.

\section{A Equação de Poisson}

Considere um domínio bidimensional que representa um campo térmico ou mecânico em estado de equilíbrio, cujo meio possui propriedades homogêneas e isotrópicas e nele existem fontes, sorvedouros ou ações externas que atuam diretamente no domínio. Considerando $u(X)$ a variável potencial escalar, a equação diferencial associada a este problema é a Equação de Poisson, em notação indicial dada por:

$$
u(X){ }_{, i i}=p(X)
$$

As condições de contorno essenciais e naturais e são definidas, respectivamente, pelas seguintes equações:

$$
u(X)=\bar{u}(X), \quad \text { em } \quad \Gamma_{u} ; \text { e } u, n_{i}(X)=\bar{q}(X), \quad \text { em } \quad \Gamma_{q}
$$

Onde $n_{i}$ é o vetor normal unitário externo ao contorno no ponto e $\Gamma=\Gamma_{\mathrm{u}}+\Gamma_{\mathrm{q}}$.

\section{Expressão do Método dos Resíduos Ponderados}

O MRP associado à Equação de Poisson pode ser apresentado em geral de acordo com a seguinte equação integral [3]:

$$
\begin{array}{r}
\int_{\Gamma u}[\bar{u}(X)-u(X)] q *(\xi ; X) d \Gamma+\int_{\Gamma q}[q(X)-\bar{q}(X)] u *(\xi ; X) d \Gamma= \\
\int_{\Omega}[p(X)-u, i i(X)] u *(\xi ; X) d \Omega
\end{array}
$$

$\mathrm{Na}$ Equação (3) as funções de ponderação são: a solução fundamental do problema de Poisson $u^{*}(\xi, X)$, considerando um meio homogêneo infinito sujeito a uma ação concentrada no ponto $\xi$, denominado fonte; e $q^{*}(\xi, X)$ é sua derivada normal ao contorno externo $\Gamma$. Por serem funções solução de um problema correlato, constroem um espaço solução afim e sua potencialidade na minimização de resíduos é elevada. Ainda nesta equação, $u(X)$ deve ser interpretado como uma solução aproximada da solução exata, dada pela equação (1). A equação (3) inclui a minimização de todos os tipos de resíduos que podem aparecer nas aproximações da solução, seja no domínio $\Omega$ e nas fronteiras $\Gamma_{u}$ e $\Gamma_{\mathrm{q}}$.

Uma vez que $p(X)$ é uma função conhecida, nos procedimentos clássicos do MEC 
opera-se matematicamente o lado esquerdo da equação (3), juntamente com a integral de domínio referente ao Laplaciano do potencial $u(X)$, visando obter uma expressão adequada à aplicação imediata dos procedimentos típicos de discretização do MEC. Com esse propósito, é preciso realizar operações matemáticas que incluem duas integrações por partes e utilização do Teorema da Divergência [1]. Feito isso, estabelece-se a forma integral denominada inversa, equivalente a equação (4), dada por:

$$
c(\xi) u(\xi)+\int_{\Gamma} u(X) q^{*}(\xi ; X) d \Gamma-\int_{\Gamma} q(X) u *(\xi ; X) d \Gamma=\int_{\Omega} p(X) u *(\xi ; X) d \Omega
$$

O coeficiente $\mathrm{c}(\xi)$ depende do posicionamento do ponto $\xi$ com relação ao domínio físico $\Omega(X)$ e procedimentos matemáticos relativamente elaborados demonstram que, por exemplo, para contornos suaves seu valor é igual a meio [2].

Para problemas em que as ações de domínio são harmônicas, ou seja:

$$
p(X),_{i i}=0
$$

é possível empregar o procedimento que usa o chamado Tensor de Galerkin $G^{*}(\xi ; X)$ [2] e, a menos dos erros de discretização, integrar exatamente o lado direto da equação (4), sem que hajam resíduos a serem minimizados com relação a função $p(X)$. Neste caso, tem-se:

$$
\begin{aligned}
& \int_{\Omega} p(X) u^{*}(\xi ; X) d \Omega=\int_{\Omega} p(X) G^{*},{ }_{i i}(\xi ; X) d \Omega= \\
& \int_{\Gamma} p(X) G^{*},{ }_{i}(\xi ; X) n_{i}(X) d \Gamma-\int_{\Gamma} p,{ }_{i}(X) n_{i}(X) G^{*}(\xi ; X) d \Gamma
\end{aligned}
$$

\section{Procedimento Recursivo}

A ideia do processo recursivo é aplicar a sentença integral de contorno com pontos fonte localizados em locais diferentes daqueles que geraram os nós de fronteira originais. A expectativa é a obtenção de resultados com uma melhor precisão, devido à correlação entre a reaplicação da equação integral de contorno e uma nova minimização de erros. Vale ressaltar que a equação integral de contorno (4) é matematicamente equivalente a uma expressão do Método dos Resíduos Ponderados (MRP). Assim, caso não sejam introduzidas aproximações adicionais na representação das ações de domínio, a substituição da equação (6) na equação (4) não altera o entendimento de que a escolha de novos pontos fonte no contorno represente uma nova minimização dos resíduos de integração sobre o domínio e o contorno.

\section{Testes numéricos}

\subsection{Primeiro teste: barra sujeita a uma ação de domínio constante}


A Figura 1 mostra as características físicas e geométricas do primeiro exemplo. O comprimento é L, a massa específica é po, o módulo de Young é E e a força de corpo é g. Para simplificar, todas estas variáveis são tomadas igual à unidade. A equação de governo para este problema é dada por:

$$
\frac{d^{2} u}{d x_{1}^{2}}=\frac{\rho_{0} g}{E}
$$

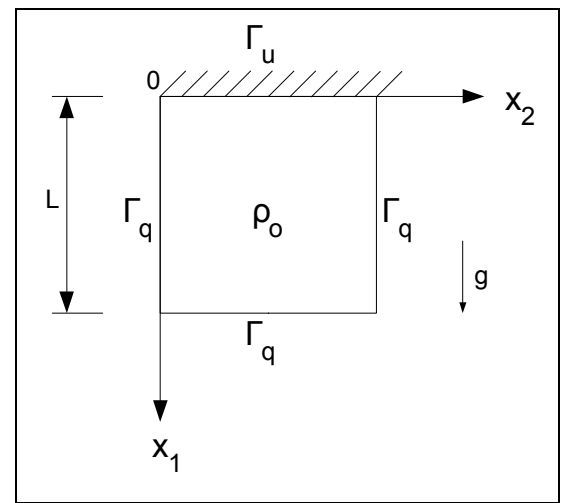

Figura 1: Barra sujeita a uma ação de domínio constante.

Diferentes malhas com elementos de contorno lineares são empregadas. Nos cantos são usados nós duplos. Para o procedimetnos recursivo, os novos pontos fonte são tomados centralizados em cada elemento. Analogamente ao procedimento direto do MEC, valores recursivos de deslocamento e forças de superfície são calculados nos elementos em que não foram prescritas tais condições.

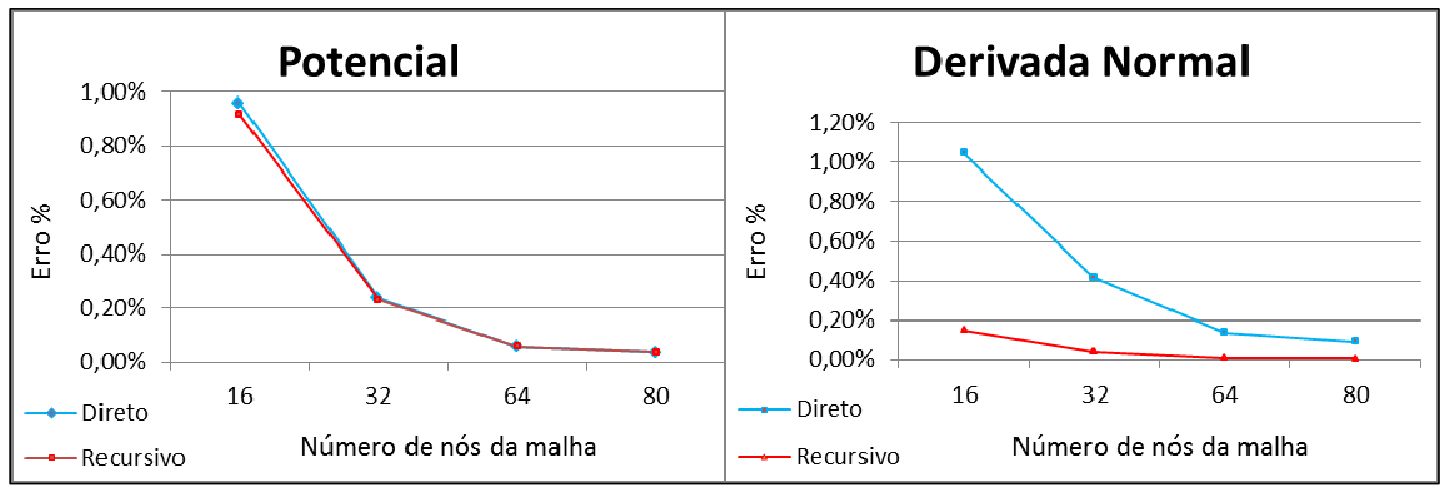

Figura 2: (a) Erro percentual dos valores do potencial; (b) Erro percentual dos valores da derivada normal.

A Figura 2a mostra os resultados obtidos para os deslocamentos recursivos em 
comparação com os valores diretos. Percebe-se o melhor desempenho deste último, particularmente nas malhas menos refinadas. Para as forças de superfície, de maior dificuldade numérica em qualquer método gerado a partir de variáveis primitivas (como o deslocamento, neste caso) o desempenho do procedimento recursivo foi bem superior (Figura 2b), atestando a realidade do procedimento de reminimização de resíduos.

\subsection{Segundo teste: membrana sob ação de carga constante}

Este caso bidimensional pode ser fisicamente interpretado como uma membrana com suas extremidades fixas sujeita a um carregamento constante em seu domínio, dado por:

$$
\frac{\partial^{2} u}{\partial x_{1}^{2}}+\frac{\partial^{2} u}{\partial x_{2}^{2}}=p
$$

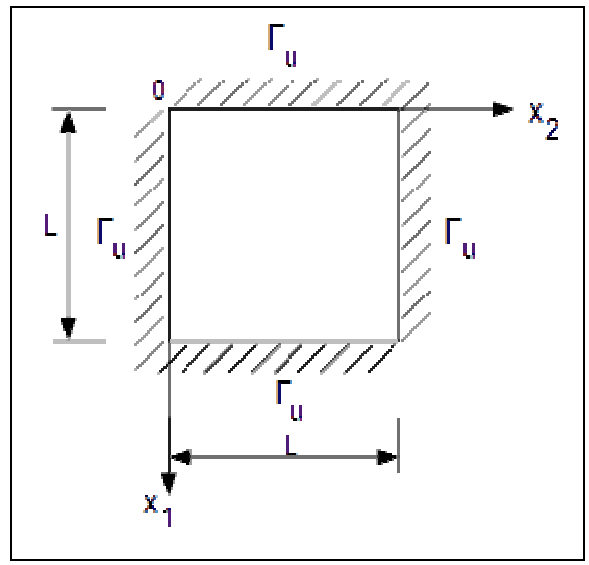

Figura 3: Membrana fixa no contorno sujeita a uma ação de domínio constante.

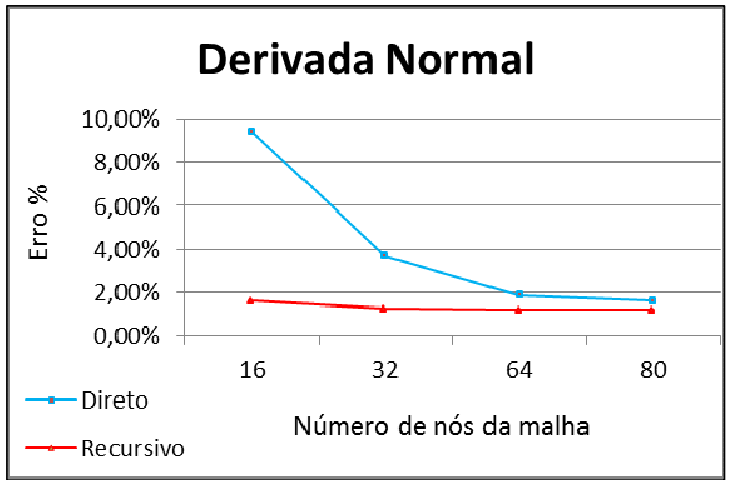

Figura 4: Erro percentual dos valores da derivada normal.

Neste problema apenas as forças de superfície são calculadas, visto que os 
deslocamentos no contorno são prescritos. Os resultados são apresentados na Figura 4. Pode-se perceber que neste caso, mais complexo, também houve redução dos erros nos valores de contorno, sendo ainda mais significativo o desempenho do procedimento recursivo quando malhas menos refinadas são utilizadas.

\section{Conclusões}

Sabe-se que a utilização de uma equação integral de contorno para determinar os valores internos produz resultados numéricos com maior precisão do que nos pontos de contorno. Isto pode ser justificado por uma nova minimização de erros imposta pela reaplicação da sentença de resíduos ponderados.

Tal como nos problemas de Laplace, no caso dos deslocamentos uma minimização eficaz dos resíduos pode ser alcançado apenas para malhas pouco refinadas, em que se pode perceber que a solução numérica ainda não havia representado adequadamente o campo proposto. Neste sentido, verifica-se que a diferença nos resultados entre as soluções direta e recursiva se reduziu bastante com o refinamento da malha.

No que se refere às forças de superfície, o procedimento recursivo tem mostrado um desempenho bem melhor, e a razão para isto deve se relacionar a maior dificuldade que os métodos numéricos apresentam para aproximar grandezas derivadas da variável primal. Este desempenho é ainda mais pronunciado em casos que apresentam descontinuidades e singularidades.

O custo computacional do procedimento recursivo não é alto porque nenhum novo sistema de equações precisa ser resolvido. Os níveis de erro foram similares àqueles encontrados nas simulações dos problemas de Laplace, o que credencia a aplicação da técnica em problemas mais elaborados, como os governados pelas equações de Helmholtz e Navier, esta última referente a problemas de campo vetorial.

\section{Referências}

[1] C. A. Brebbia, The Boundary Element Method for Engineers, Pentech Press, (1978).

[2] C. A. Brebbia, J.C. Telles and L.C. Wrobel, Boundary Element Techniques, Springer Verlag (1984).

[3] C. A. Brebbia and S. Walker, Boundary Element Techniques in Engineering, NewnesButterworths, London, (1980).

[4] C. F. Loeffler, A recursive application of the integral equation in the boundary element method, Engineering Analysis with Boundary Elements, 35, 77-84, (2011).

[5] J. N. Reddy, An Introduction to the Finite Element Method, McGraw-Hill, (1993). 